\title{
Fuzzy Petri Net Model for Dynamic Alert Management System
}

\author{
S.Jayasudha \\ Depatment of Mathematics \\ Bharath University \\ Chennai -73
}

\author{
A.Ammu Qudsiya \\ Department of Mathematics \\ Agni College of Engineering \\ and Technology, Chennai
}

\author{
K.Ramanathan \\ Department of Mathematics \\ KCG college of Engineering \\ Chennai
}

\begin{abstract}
A Dynamic alert management system in Industrial Process based on fuzzy petri nets is presented in this paper. By utilizing fuzzy petri nets, a technique based fuzzy rules algorithm is developed in alert management system. This algorithm is helpful in illustrating how fuzzy petri nets describe actions to be performed in this system. In this paper, the existing procedure scheme for dynamic alert system has been transformed into an equivalent rule base system.
\end{abstract}

\section{Keywords}

Fuzzy Petri nets, Petrinets, Alert management system, fuzzy rules, Reachability graph

\section{INTRODUCTION}

Parallel or Distributed systems are common in Real time applications. Petri nets are used for specifying the behavior and establish the correctness of the same.[1] .

Alarm management becomes more important for industrial process. The basic purpose of alarm annunciation is to alert the operator to abnormal operating situations. The ultimate objective is to prevent, or at least minimize, physical and economic loss through operator intervention in response to the condition that was alarmed. The main problem with basic alarm management is that these features are static.

The primary purpose of dynamic alarm annunciation is to alert the operator to relevant abnormal operating situations. Dynamic alarm annunciation management focuses the operator's attention by eliminating extraneous alarms, providing better recognition of critical problems, and insuring swifter, more accurate operator response.

Conventional modeling provides limited scope for modeling such complex alert management scenarios with multiple interdependencies. Alert management involves retention of earlier states of the alerts and also to be able to prevent repeated alerts based on specific situations. Hence the chosen modeling tool should allow depiction of interdependencies, cause- effect relationships, conditional branching, hierarchical representations etc., for successful modeling. This paper is a novel effort to show how fuzzy Petri nets can be used to model such dynamic environments.

The aim of this paper is to develop a framework and modeling approach for an alert management and dynamic system using fuzzy petri nets. In this paper, the description of this application by constructing the FPN model in the following section, Fuzzy Petri nets and mapping fuzzy rules to fuzzy petri net $[1,2,8,9]$ is introduced. Next section deals with modeling of dynamic alarm management using Fuzzy Petri nets $[3,4,6]$ as application found in[5,7] specified in algorithmic format. In this paper it is obtained the Rule base format that can be implemented by fuzzy petri nets.

Alert management is one of the key activities in many critical applications. Alert Management involves the activities based on the raised alerts. Alert suppression is the term used to avoid alerts either altogether or based on some special conditions like repeated alerting etc.,

\section{FUZZY PETRI NETS}

In this section ,the combination of petri nets and fuzzy logic in order to extract both advantages namely, concurrency and conceptualizing the impreciseness is considered.

\subsection{Fuzzy Petri Nets for Fuzzy Rules}

The following fuzzy petri net (FPN) structure to model the fuzzy rules is introduced:

\section{(P, $\left.\mathrm{P}_{\mathrm{s}}, \mathrm{P}_{\mathrm{c}}, \mathrm{T}, \mathrm{TF}, \mathrm{TRTF}, \mathrm{A}, \mathrm{I}, \mathrm{O}, \mathrm{TT}, \mathrm{TTF}, \mathrm{AEF}, \mathrm{PR}, \mathrm{PPM}, \mathrm{TV}\right)$} Where

(i) $\quad \mathrm{P}$ is a finite set of fuzzy places, in which

- $\quad \mathrm{P}_{\mathrm{s}} \subset \mathrm{P}$ is a finite set of input places for primitive events or conditions.

- $\quad \mathrm{P}_{\mathrm{c}} \subset \mathrm{P}$ is a finite set of output places for actions or conclusions.

(ii) $\quad \mathrm{T}$ is a finite set of transitions.

(iii) $\quad \mathrm{A} \subset(\mathrm{P} X \mathrm{~T} \cup T X P)$ is a finite set of arcs for connections between places and transitions.

(iv) TT is a finite set of token types.

(v) TTF: $\mathrm{P} \rightarrow \mathrm{TT}$ is token type function, mapping each fuzzy place $\epsilon$ P to token type $\epsilon$ TT.

(vi) $\quad$ AEF: Arc $\rightarrow$ expression, is arc expression function mapping each arc to an expression which carries the information (token values).

(vii) PR is a finite set of propositions, corresponding to either events or conditions or actional conclusions.

(viii) $\quad$ PPM: $\mathrm{P} \rightarrow \mathrm{PR}$, is place to proposition mapping, where $|P R|=|P|$.

(ix) $\quad \mathrm{TV}: \mathrm{P} \rightarrow[0,1]$ is truth values of proposition assigned to places.

Hence FPN is a directed graph with edges indicating the linking the places to transitions and transitions to places. Similar to the conventional petri nets except the nodes either places or transitions are associated with fuzzy values.

\subsection{Fuzzy Production Rules}

Let $\mathrm{R}$ be a set of fuzzy production rules:

$\mathrm{R}=\{\mathrm{R} 1, \mathrm{R} 2, \ldots . \mathrm{Rm}\}$, and a fuzzy production rule Ri us shown as follows 
$\mathrm{Ri}:$ If $\mathrm{dj}$ then $\mathrm{dk},(\mathrm{CF}=\mu \mathrm{i})$

IF all propositions in the antecedent dj have value true THEN the propositions in the consequent $\mathrm{dk}$ are true.

Where $\mathrm{di}=\{\operatorname{dj} 1, \operatorname{dj} 2, \ldots . \operatorname{djn}\}$, represents the antecedent part which comprises of one or more propositions connected by either "AND" or "OR" in the rule;

$\mathrm{Dk}=\{\mathrm{dk} 1, \mathrm{dk} 2, \ldots . \mathrm{dkn}\}$ represents the consequent part which comprise $3 \mathrm{~s}$ of one or more propositions connected by "AND" in the rule;

$\mu \mathrm{i}$ denotes the certainty factor $(\mathrm{CFi})$ of the rule $\mathrm{Ri}$. Generally, FPRs are classified into four types as follows:

Type 1: IF dj , THEN dk , $(\mathrm{CF}=\mu)$,

Type 2: IF dj1 and dj2 and .....and djn THEN dk $(\mathrm{CF}=\mu)$,

Type 3: IF dj1 or dj2 or .....or djn THEN dk $(\mathrm{CF}=\mu)$,

Type 4: IF dj THEN dk1 and dk2 and ......and dkn $(\mathrm{CF}=\mu)$,

FPN models of the 4 types of composite fuzzy production rules.

\subsection{Constructing the Fuzzy Petri net}

FPN is constructed directly from given rule definitions. Construction algorithm takes the rules specified in a predefined syntax as input. Then it generates the corresponding FPN structure automatically. More Specifically, first the rule definitions are obtained from the user. For each rule, a rule object is created, and the event, condition and action parts of the rule are examined. In that, related FPN places such as primitive events, fuzzy primitive events, etc., are created. Then, the fuzzy inference groups which are the concurrent rule sets that get triggered at the same time, are determined. Finally transitions are constructed over these FPN places.

\section{FORMALISM OF A DYNAMIC ALERT MANAGEMENT SYSTEM}

Alerts have been used in different forms since ancient days starting from sound signals to signal flares etc., Alert were used to bring to attention some event or some happening in order to avoid or react to that situation. Traditional alerts have been very simple and easy to use, but with the evolution of technology and maximization of automation across industries and applications, alert have become more and more complex.

In this section, we provide a Fuzzy Petri net based representation of the concept above. The objective of the work in this section is to discuss how fuzzy petri nets, a technique based fuzzy rules algorithm is developed in alert management system.

We have taken a system which monitors the temperature of an industrial Boiler.[6]. An on-off controller is the simplest form of temperature control device. The output from the device is either on or off, with no middle state. An on-off controller will switch the output only when the temperature crosses the set point. For heating control, the output is on when the temperature is below the set point, and off above the set point.
Since the temperature crosses the set point to change the output state, the process temperature will be cycling continually, going from below set point to above, and back below. In cases where this cycling occurs rapidly, and to prevent damage to contractors and valves, and on-off differential, or "hysteresis".is added to the controller operations.

One special type of on-off control used for alarm is a limit controller. This controller uses a latching relay, which must be manually reset, and is used to shut down a process when a certain temperature is reached.

The example taken for the case here will show how the annoying redundant alert can be effectively managed using a suppression mechanism. We have taken a system which monitors the temperature of an industrial boiler. If the boiler's internal temperature exceeds a specific limit an alarm will be raised and monitoring of the temperature will continue. If the temperature does not exceed the set limit, then also the monitoring will be continued with the alarm in the switch off mode. If the alarm is in the switch on mode, still the monitoring will be continued, but the redundant alarming will be avoided. This is done using inhibitor arcs.

The status of the alarm depends upon the limit temperature(LT) and recorded temperature(RT). State of the alarm changes from off to on mode if the following inequality holds good.

LT $-\mathrm{RT}<0$. State of the alarm changes from on to off mode if the following inequality holds good. LT $-\mathrm{RT} \geq 0$. The parameter taken here is temperature.

Table 1. Description of Control Variables

\begin{tabular}{|l|l|l|}
\hline S.No. & Control Variables & Symbols \\
\hline 1. & Limit temperature & LT \\
\hline 2. & Recorded temperature & RT \\
\hline 3. & Switch ON & 1 \\
\hline 4. & Switch off & 0 \\
\hline 5. & Alert on & 1 \\
\hline 6. & Alert off & 0 \\
\hline 7. & Monitoring break on & $\mathrm{Mb}=1$ \\
\hline 8. & Monitoring break off & $\mathrm{Mb}=0$ \\
\hline
\end{tabular}




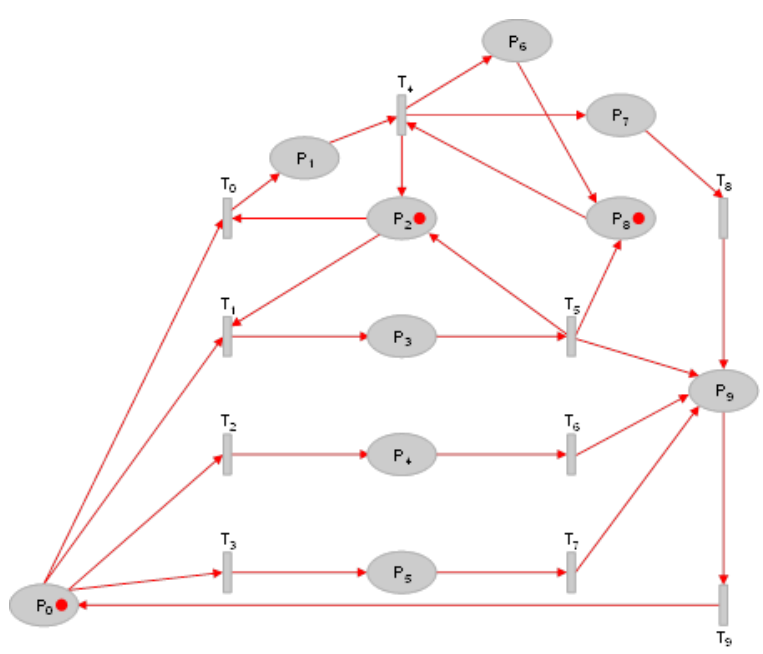

Fig.1 Fuzzy Petri net Model of Dynamic Alert

\section{ALGORITHM FOR THE ACTION PERFORMED}

STEP 1: IF $\mathrm{P}_{0}$,, $\left.\mathrm{RT}>\mathrm{LT}\right)$ THEN $\mathrm{P}_{1}$ is true

STEP 2: IF $\mathrm{P}_{1}$ holds THEN $\mathrm{P}_{2}$ is true

STEP 3: If $\mathrm{P}_{3}$, (switch==0) THEN $\mathrm{P}_{8}$ is true,

$($ alert $=0)$

STEP 4: If $\mathrm{P}_{8},($ switch $==0)$ THEN $\mathrm{P}_{7}$ is true $($ alert $=1)$

STEP $5 \operatorname{IF~P}_{7},($ alert $==1)$ THEN $\mathrm{P}_{9}$ holds $(\mathrm{Mb}=1)$

STEP 6: IF $\mathrm{P}_{9},(\mathrm{Mb}==1)$ THEN $\mathrm{P}_{0}$ holds

STEP 7: IF $\mathrm{P}_{0},(\mathrm{RT}<\mathrm{LT})$ THEN $\mathrm{P}_{3}$ is true

$($ switch $=0)$

STEP 8: IF $\mathrm{P}_{9},(\mathrm{Mb}==1)$ THEN $\mathrm{P}_{0}$ holds .

STEP 9: IF $\mathrm{P}_{0}$ is in alert mode THEN $\mathrm{P}_{4}$ is true.

STEP 10: IF $\mathrm{P}_{4}$, alert mode is in sleep mode THEN $\mathrm{P}_{9}$ holds $(\mathrm{Mb}=1)$

STEP 11: IF $\mathrm{P}_{9},(\mathrm{Mb}=1)$ THEN $\mathrm{P}_{0}$ is true

STEP 12: IF $\mathrm{P}_{0}$ is in normal mode THEN $\mathrm{P}_{5}$ holds

STEP 13: IF $\mathrm{P}_{5}$, normal mode is in sleeping mode THEN $\mathrm{P}_{9}$ holds $(\mathrm{Mb}=1)$.

STEP 14: IF $\mathrm{P}_{9}(\mathrm{Mb}=1)$ THEN $\mathrm{P}_{0}$ is true.

\section{ANALYSIS OF FUZZY PETRI NETS}

This section describes how fuzzy petri nets can be analyzed. Similar analysis[8] is used. Two major Petri net analysis methods, the cover ability tree and state equation are used to analyze fuzzy petri nets.

\subsection{The Cover Ability Tree}

The cover ability tree represents the reachability set of a fuzzy petri net. Given a fuzzy Petri net a tree representation of the markings can be constructed. In this tree, a symbol w is used to represent " infinity", nodes represent markings reachable from $\mathrm{M}_{0}$ and each are represents an uncertain transition firing that transforms one marking to another. Some of the behavioral properties that can be studied by using the cover ability tree are bounded ness, safeness and deadlock in uncertain transitions. For a bounded fuzzy Petri net, the cover ability tree is called the reachability tree.

\subsection{State Equation}

The state equation that governs the dynamic behavior of concurrent fuzzy systems modeled by fuzzy Petri nets is represented by $A^{T} x=\Delta M$ where $\Delta M=M_{n}-M_{0}$. A is the incidence matrix and $\mathrm{x}$ is an $\mathrm{n} x 1$ column vector called firing count vector. The $i^{\text {th }}$ entry of $x$ denoted as the number of times that uncertain transition $t_{i}$ must fire to transform $M_{0}$ to $M_{n}$. The state equations is used to solve the reachability problem.

\subsection{INCIDENCE MATRIX}

Table 4. Pre- Incidence Matrix

\begin{tabular}{|l|l|l|l|l|l|l|l|l|l|l|}
\hline & $\mathrm{T}_{0}$ & $\mathrm{~T}_{1}$ & $\mathrm{~T}_{2}$ & $\mathrm{~T}_{3}$ & $\mathrm{~T}_{4}$ & $\mathrm{~T}_{5}$ & $\mathrm{~T}_{6}$ & $\mathrm{~T}_{7}$ & $\mathrm{~T}_{8}$ & $\mathrm{~T}_{9}$ \\
\hline $\mathrm{P}_{0}$ & 1 & 1 & 1 & 1 & 0 & 0 & 0 & 0 & 0 & 0 \\
\hline $\mathrm{P}_{1}$ & 0 & 0 & 0 & 0 & 1 & 0 & 0 & 0 & 0 & 0 \\
\hline $\mathrm{P}_{2}$ & 0 & 1 & 0 & 0 & 0 & 0 & 0 & 0 & 0 & 0 \\
\hline $\mathrm{P}_{3}$ & 0 & 0 & 0 & 0 & 0 & 1 & 0 & 0 & 0 & 0 \\
\hline $\mathrm{P}_{4}$ & 0 & 0 & 0 & 0 & 0 & 0 & 1 & 0 & 0 & 0 \\
\hline $\mathrm{P}_{5}$ & 0 & 0 & 0 & 0 & 0 & 0 & 0 & 1 & 0 & 0 \\
\hline $\mathrm{P}_{6}$ & 1 & 0 & 0 & 0 & 0 & 1 & 0 & 0 & 0 & 0 \\
\hline $\mathrm{P}_{7}$ & 0 & 0 & 0 & 0 & 0 & 0 & 0 & 0 & 1 & 0 \\
\hline $\mathrm{P}_{8}$ & 0 & 1 & 0 & 0 & 1 & 0 & 0 & 0 & 0 & 0 \\
\hline $\mathrm{P}_{9}$ & 0 & 0 & 0 & 0 & 0 & 0 & 0 & 0 & 0 & 1 \\
\hline
\end{tabular}

Table 5. Post- Incidence Matrix

\begin{tabular}{|l|l|l|l|l|l|l|l|l|l|l|}
\hline & $\mathrm{T}_{0}$ & $\mathrm{~T}_{1}$ & $\mathrm{~T}_{2}$ & $\mathrm{~T}_{3}$ & $\mathrm{~T}_{4}$ & $\mathrm{~T}_{5}$ & $\mathrm{~T}_{6}$ & $\mathrm{~T}_{7}$ & $\mathrm{~T}_{8}$ & $\mathrm{~T}_{9}$ \\
\hline $\mathrm{P}_{0}$ & 0 & 0 & 0 & 0 & 0 & 0 & 0 & 0 & 0 & 1 \\
\hline $\mathrm{P}_{1}$ & 1 & 0 & 0 & 0 & 1 & 0 & 0 & 0 & 0 & 0 \\
\hline $\mathrm{P}_{2}$ & 0 & 0 & 0 & 0 & 1 & 1 & 0 & 0 & 0 & 0 \\
\hline $\mathrm{P}_{3}$ & 0 & 1 & 0 & 0 & 0 & 0 & 0 & 0 & 0 & 0 \\
\hline $\mathrm{P}_{4}$ & 0 & 0 & 1 & 0 & 0 & 0 & 0 & 0 & 0 & 0 \\
\hline $\mathrm{P}_{5}$ & 0 & 0 & 0 & 1 & 0 & 0 & 0 & 0 & 0 & 0 \\
\hline $\mathrm{P}_{6}$ & 0 & 0 & 0 & 0 & 1 & 0 & 0 & 0 & 0 & 0 \\
\hline $\mathrm{P}_{7}$ & 0 & 0 & 0 & 0 & 1 & 0 & 0 & 0 & 0 & 0 \\
\hline $\mathrm{P}_{8}$ & 0 & 1 & 0 & 0 & 0 & 1 & 0 & 0 & 0 & 0 \\
\hline $\mathrm{P}_{9}$ & 0 & 0 & 0 & 0 & 0 & 1 & 1 & 1 & 1 & 0 \\
\hline
\end{tabular}


Table 6. Combined Matrix

\begin{tabular}{|l|l|l|l|l|l|l|l|l|l|l|}
\hline & $\mathrm{T}_{0}$ & $\mathrm{~T}_{1}$ & $\mathrm{~T}_{2}$ & $\mathrm{~T}_{3}$ & $\mathrm{~T}_{4}$ & $\mathrm{~T}_{5}$ & $\mathrm{~T}_{6}$ & $\mathrm{~T}_{7}$ & $\mathrm{~T}_{8}$ & $\mathrm{~T}_{9}$ \\
\hline $\mathrm{P}_{0}$ & -1 & -1 & -1 & -1 & 0 & 0 & 0 & 0 & 0 & 1 \\
\hline $\mathrm{P}_{1}$ & 1 & 0 & 0 & 0 & -1 & 0 & 0 & 0 & 0 & 0 \\
\hline $\mathrm{P}_{2}$ & 0 & -1 & 0 & 0 & 1 & 1 & 0 & 0 & 0 & 0 \\
\hline $\mathrm{P}_{3}$ & 0 & 1 & 0 & 0 & 0 & -1 & 0 & 0 & 0 & 0 \\
\hline $\mathrm{P}_{4}$ & 0 & 0 & 1 & 0 & 0 & 0 & -1 & 0 & 0 & 0 \\
\hline $\mathrm{P}_{5}$ & 0 & 0 & 0 & 1 & 0 & 0 & 0 & -1 & 0 & 0 \\
\hline $\mathrm{P}_{6}$ & 1 & 0 & 0 & 0 & 1 & -1 & 0 & 0 & 0 & 0 \\
\hline $\mathrm{P}_{7}$ & 0 & 0 & 0 & 0 & -1 & 0 & 0 & 0 & -1 & 0 \\
\hline $\mathrm{P}_{8}$ & 0 & 1 & 0 & 0 & -1 & 1 & 0 & 0 & 0 & 0 \\
\hline $\mathrm{P}_{9}$ & 0 & 0 & 0 & 0 & 0 & 1 & 1 & 1 & 1 & -1 \\
\hline
\end{tabular}

\subsection{Reachability Graph}

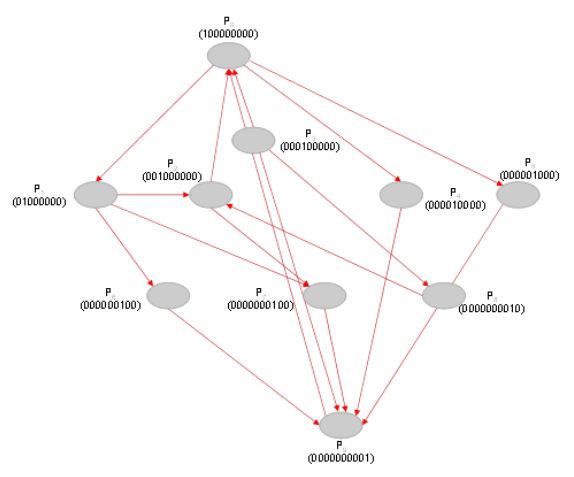

As a result it can be conclude that the net is bounded.[5,9]

\section{CONCLUSION}

In this paper, it has been emphasized the importance of Alert Management in an alarm system. By utilizing fuzzy Petri nets, a technique based on fuzzy rules states is developed in alert management system. In Fig. 2 the petri net shown has only the nodes labeled with binary sequence indicating the firing configurations'. It follows from [9].

\section{REMARK}

As per theorem in [9], here we established the case by our example. In future this can be extended to Dynamic Alert System.

As the future scope, this representation can be automated or evaluated by an appropriate Petri net tool. Moreover Tsunami
Alert system may be designed and developed similar as a case study similar to the one we have done.

\section{ACKNOWLEDGEMENTS}

The author's thanks for the Referee's Comments

\section{REFERENCES}

[1] Bidyut Biman Sarkar Nabendu Chaki, “ Transaction Management for Distributed Database Using petri Nets" , International Journal of Computer Information Systems and Industrial Management Applications, ISSN: 2150 - 7988 Vol.2 (2010) pp. 069 076.

[2] B.Bostan-Korperglu,A.Yazici, " An active object oriented database approach, in; Proceedings of $13^{\text {th }}$ IEEE International conference on fuzzy system(FUZZIEEE),Budapest,Hungary,25-29,July 2004.

[3] Burcin Bostan ,-Korpeoglu,Adnan Yaziici, “A fuzzy Petri net model for intelligent databases", Data \& knowledge Engineering, 62(2007)219247.

[4] Jonathan Lee, “ Fuzzy Petri nets for modeling Rule-Based Reasoning", International Journal on Artificial Intelligence Tools, Vol.O.No.O(1998)000-000@world Scientific Publishing Company.

[5] A.Ammu Qudsiya and K.Rangarajan, "Dynamic Alert Management System Using Timed Petri Nets" Vol 03, Issue 01; January-April 2012 Research Journal of Computer Systems Engineering - RJCSE http://technicaljournals.org cFACT -2012, Loyola College, Chennai, TN ISSN: 2230-8563; e-ISSN-22308571 .

[6] S.Jayasudha,K.Ramanathan, K.Rangarajan,'Fuzzy Petri nets and Fuzzy Cognitive Maps", International Journal of Computer Application, 46(11):5-9, May 2012. Published by Foundation of Computer Science, New York, USA.

[7] Introduction to Temperature Controller, www.omega.com

[8] Burcin Bostan ,-Korpeoglu,Adnan Yaziici ,’Using Fuzzy Petri nets for Static Analysis of Rule-Bases",pp7281,2004,Springer-Verlag Berlin Heidelberg 2004.

[9] Tiehua Cao and Arthur C,Sanderson, " Variable Reasoning and Analysis about Uncertainty with Fuzzy Petri Nets" 